\title{
ANALYSIS OF THE 1980 NOVEMBER 18 LIMB FLARE OBSERVED BY THE HARD X-RAY IMAGING SPECTROMETER (HXIS)
}

\author{
E. Haug, ${ }^{*}$ G. Elwert* and P. Hoyng** \\ *Lehrstuhl für Theoretische Astrophysik, Universität Tübingen, \\ D-7400 Tübingen, F.R.G. \\ ** Laboratory for Space Research, 3527 HS Utrecht, \\ The Netherlands
}

\section{ABSTRACT}

\begin{abstract}
X-ray images of the 18 November 1980 limb flare taken by the HXIS instrument aboard SMM were analysed. The hard X-rays originated from three spots on the SW limb of the solar disk with different altitudes and time evolution. The locations of the brightest spots in hard and soft $x$-rays are compared with the predictions of flare models. The X-ray spctra from the pixels with highest count rates can be fitted by power laws. The spatial variation of the spectral index is in agreement with the existence of a non-thermal electron component.
\end{abstract}

For the further development of the physics of solar flares it is important to obtain information about the height distribution of solar X-ray emission. We therefore selected from the HXIS/1/ data a limb flare which occurred on 18 November 1980 at 14:51 UT in Active Region No. 2779 (spot group 255) directly

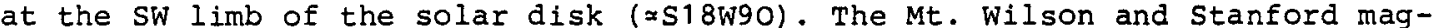
netograms taken two days earlier show an extended bipolar field configuration associated with the spot group 255. There is no doubt that the preceding region of negative polarity has disappeared behind the solar limb at the time of the $x$-ray burst. The impulsive burst was also recorded in hard $x-r a y s$ ( $229 \mathrm{keV}$ ) by the Hard X-Ray Burst spectrometer (HXRBS) aboard SMM /2/.

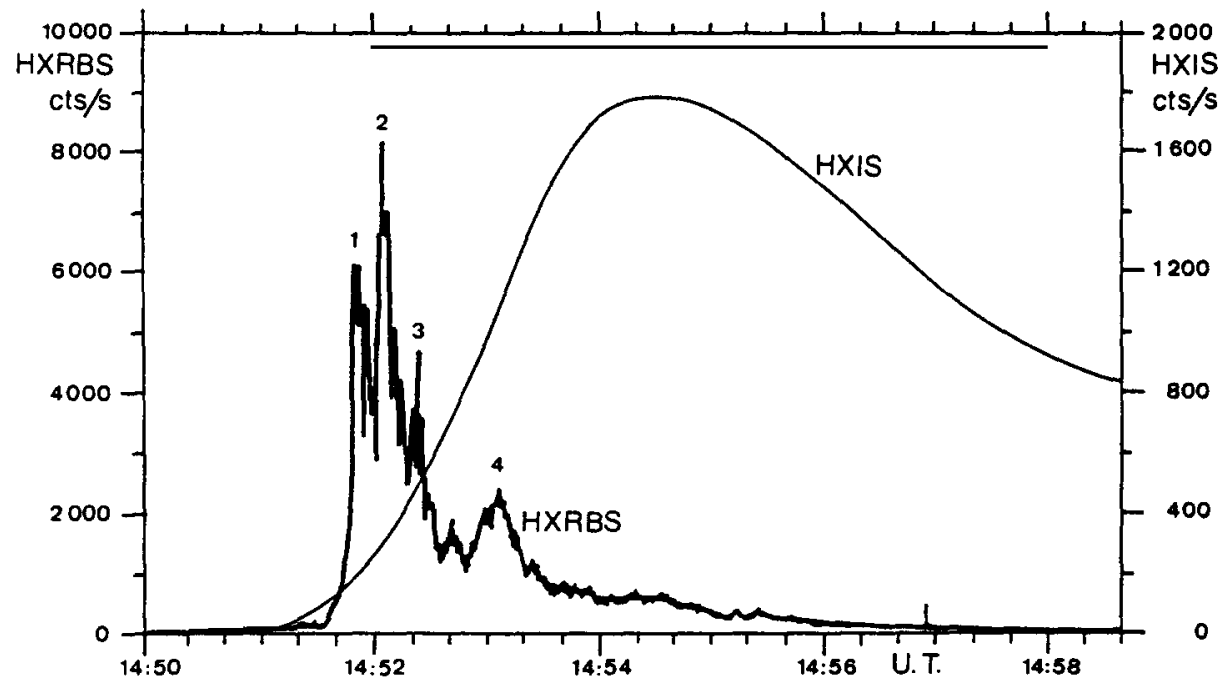

Fig. 1. Time evolution of the soft and hard $\mathrm{X}$-radiation as observed by HXIS and HXRBS, respectively.

Fig. 1 shows the temporal evolution of the soft $x$-radiation as observed by HXIS (sum of the count rates of all the six energy bands, 3.5 to $30 \mathrm{keV}$, in the coarse field of view /1// and of the hard $x$-radiation as observed by HXRBS. Here no correlation can be seen. However, in the two highest energy bands of HXIS (16-30 keV) the peak X-ray fluxes are reached at the same times as in HXRBS so that there is a distinct time behaviour of the various energy bands. The spatial resolution of HXIS ( 8 ") allows to investigate the time 
evolution of the $\mathrm{x}$-radiation originating from relatively small source areas. During the first hard $X-r a y$ bursts (labeled 1, 2, and 3 in Fig. 1) the $X-$ radiation with energies $22-30 \mathrm{keV}$ is mainly emitted from a small area $A$ ( $F$ ig. $2 a)$. Later on the count rates in area $B$ corresponding to regions higher in the solar atmosphere exceed the rates in A (Fig. 2b), and during the last hard $X$-ray spike 4 a new $X-r a y$ source in area $C$ at still higher altitudes of the solar atmosphere $(6,000$ to $10,000 \mathrm{~km})$ flares up (Fig. 2C) and decays rapidly. After 14:54 the emission in the initially brightest area $A$ is quite weak; the same holds for area $C$. The main part of the emission now originates from the intermediate area $B$.
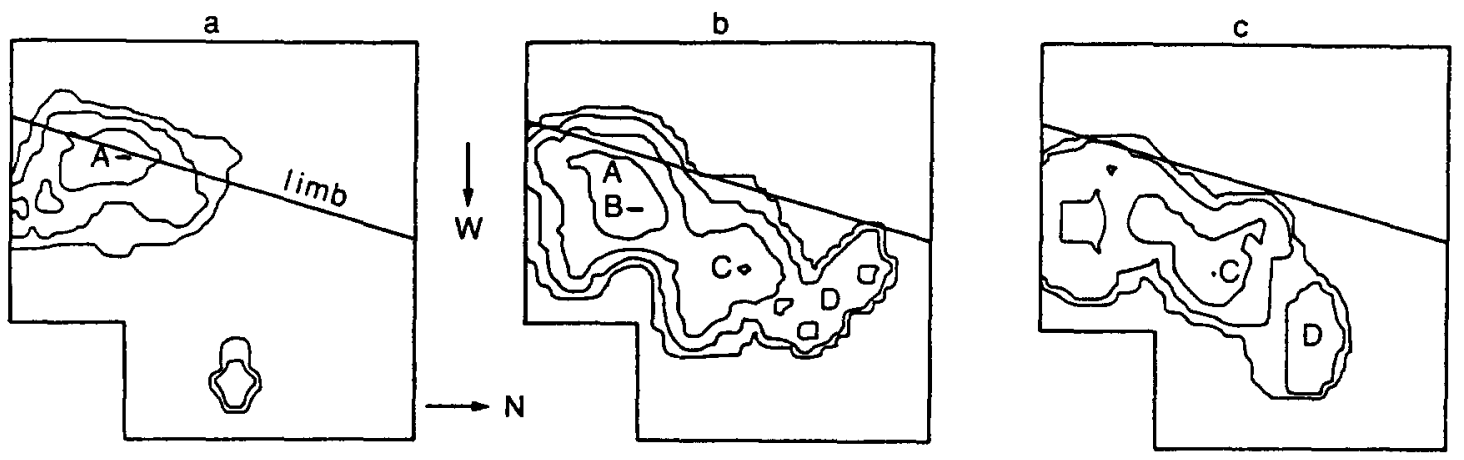

Fig. 2. 22 - $30 \mathrm{keV}$ contour maps of the flare region for the time periods (a) $14: 51: 37-14: 51: 58$, (b) $14: 52: 45-14: 53: 05$, and (c) $14: 53: 10-14: 53: 33$.

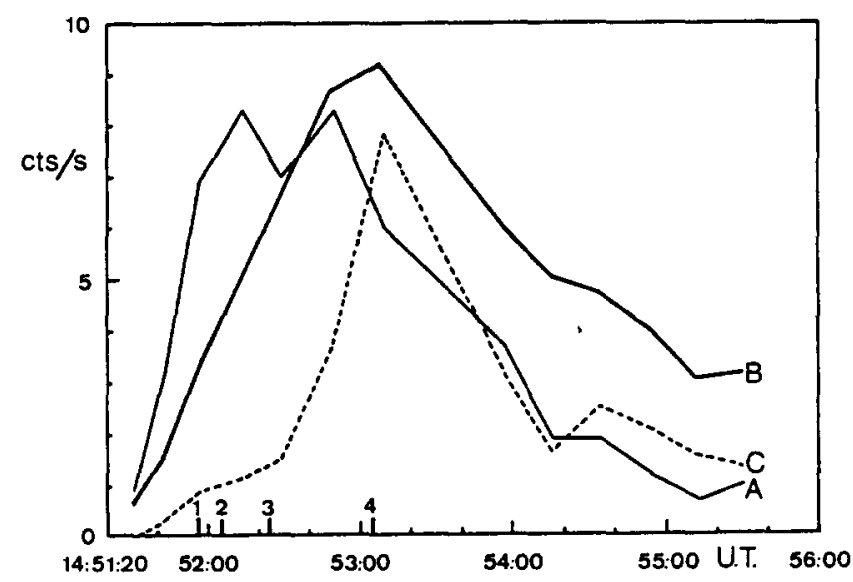

Fig. 3. Time evolution of the $22-30 \mathrm{keV}$ counting rates in the areas $A, B$, and $C$. The marks on the abscissa refer to the corresponding hard X-ray spikes of Fig. 1.

The time evolution described above is depicted in Fig. 3 showing the count rates of some selected pixels in the two highest energy bands. In order to improve statistics the count rates accumulated in 6 to 10.5 seconds of two adjacent pixels were taken together. In area $A$ the steepest $X-r$ ay enhancement occurs with peak rates between spikes 1 and 3 . In area $B$, on the other hand, the count rates increase continuously until $=14: 53$ when the last

(No. 4) of the hard X-ray bursts occurs (Fig. 1). On the sun, B corresponds to atmospheric levels which are about $6,000 \mathrm{~km}$ above $\mathrm{A}$. In the region imaged in area $C$ the first $X$-ray bursts 1 to 3 are virtually absent whereas the last spike 4 is strongly pronounced and seems to arise from this region which is situated still higher in the solar atmosphere.

In soft $X$-rays $(3.5-5.5 \mathrm{keV})$ the initial brightening starts in areas $A$ and $B$. Besides, an additional soft $X$-ray source appears in area $D$ with photon fluxes only a little less than in the main source. This pattern is sustained until 14:53:50 when the two hot spots are connected. Now the relative contribution from the initial source $A$ has considerably weakened, and the main part originates from an approximately linear strip extending from $B$ to $D$. Its length 
on the sun amounts to about $35,000 \mathrm{~km}$.

Although it is generally accepted that solar hard X-ray bursts originate as bremsstrahlung from energetic electrons, there is still much controversy as to whether these electrons obtain their energy by heating of the plasma to high temperatures $\left(z 10^{8} \mathrm{~K}\right)$ or instead form a non-thermal beam. A compromise is the dissipative thermal model /3/. In the non-thermal thick-target model both hard and soft $x$-radiation have maximum intensity at low altitudes with high plasma density. According to the dissipative thermal model the emission of soft $x$-rays originates mainly at high altitudes in the heated flare kernels whereas the high-energy emission comes from the Maxwellian tail electrons escaping to the chromosphere. Concerning the soft $x$-radiation, the observations of the 18 November 1980 limb flare do, however, not agree with this prediction: The maximum brightness at low energies appears in an extended strip approximately parallel to the solar limb with the peak flux at altitudes below $10,000 \mathrm{~km}$. At the beginning of the flare the bulk of hard $\mathrm{x}-$ radiation is emitted in the solar chromosphere. In this respect both the dissipative thermal model and the non-thermal thick-target model agree with the observations.

Further conclusions may be drawn from the spectral information provided by HXIS. Due to the low count rates the statistics are sufficiently good only in the bright pixels. Here the $\mathrm{x}$-ray spectra may be fitted to power laws. In order to be able to discriminate between flare models, it is important to know the spatial variation of the spectral index $r$. In the thick-target model $\gamma$ is expected to decrease along the direction of the injected beam. It is obvious that evidence for a beam can be furnished only during the impulsive phase(s) of an X-ray flare. Such an effect may have been observed during the first $X$-ray spike around 14:51:42 where the spectral index decreased from top to bottom in accordance with the thick-target model.

Details will be published elsewhere.

\section{REFERENCES}

1. H.F. van Beek, P. Hoyng, B. Lafleur, and G. Simnett, The Hard X-Ray Imaging Spectrometer (HXIS), Solar Phys. 65, 39 (1980)

2. L.E. Orwig, K.J. Frost, and B.R. Dennis, The Hard X-Ray Burst Spectrometer on the Solar Maximum Mission, Solar Phys. 65, 25 (1980)

3. J.C. Brown and J. Hayward, Height structure of thermal hard X-ray sources on the Sun, Solar Phys. 73, 121 (1981) 